\title{
HySenS data exploitation for urban land cover analysis
}

\author{
Fabio Dell'Acqua $\left({ }^{1}\right)$, Paolo Gamba $\left({ }^{1}\right)$, Vittorio Casella $\left({ }^{2}\right)$, Francesco Zucca $\left({ }^{3}\right)$, \\ Jon Atli Benediktsson $\left({ }^{4}\right)$, Graeme Wilkinson $\left({ }^{5}\right)$, Andre Galli $\left({ }^{6}\right)$, Eva Savina Malinverni $\left({ }^{7}\right)$, \\ Graeme Jones $\left({ }^{8}\right)$, Darrel Greenhill $\left({ }^{8}\right)$ and Lennart Ripke $\left(^{8}\right)$ \\ (') Dipartimento di Elettronica, Facoltà di Ingegneria, Università degli Studi di Pavia, Italy \\ $\left.{ }^{(}\right)$Dipartimento di Ingegneria Edile e del Territorio (DIET), Facoltà di Ingegneria, \\ Università degli Studi di Pavia, Italy \\ $\left(^{3}\right)$ Dipartimento di Scienze della Terra, Facoltà di Scienze Matematiche Fisiche e Naturali, \\ Università degli Studi di Pavia, Italy \\ $\left({ }^{4}\right)$ Department of Electrical and Computer Engineering, University of Iceland, Reyjkavik, Iceland \\ $\left(^{5}\right)$ General Faculty of Technology, Lincoln University, Lincoln, U.K. \\ $\left(^{6}\right)$ Facoltà di Agraria, Università Politecnica delle Marche, Ancona, Italy \\ ${ }^{(7)}$ Facoltà di Ingegneria, Università Politecnica delle Marche, Ancona, Italy \\ $\left.{ }^{8}\right)$ Digital Imaging Research Centre, School of Computing and Information Systems, \\ Kingston University, Surrey, U.K.
}

\begin{abstract}
This paper addresses the use of HySenS airborne hyperspectral data for environmental urban monitoring. It is known that hyperspectral data can help to characterize some of the relations between soil composition, vegetation characteristics, and natural/artificial materials in urbanized areas. During the project we collected DAIS and ROSIS data over the urban test area of Pavia, Northern Italy, though due to a late delivery of ROSIS data only DAIS data was used in this work. Here we show results referring to an accurate characterization and classification of land cover/use, using different supervised approaches, exploiting spectral as well as spatial information. We demonstrate the possibility to extract from the hyperspectral data information which is very useful for environmental characterization of urban areas.
\end{abstract}

Key words hyperspectral remote sensing - urban land use - vegetation distribution - classification

\section{Introduction}

The final aim of the work presented in this paper is to develop a methodology for the characterization of an urban environment for environmen-

Mailing address: Dr. Fabio Dell'Acqua, Dipartimento di Elettronica, Facoltà di Ingegneria, Università degli Studi di Pavia, Via Ferrata 1, 27100 Pavia, Italy; e-mail: fabio.dellacqua@unipv.it tal monitoring. This topic is extremely interesting and urgent, given the huge amount of problems in urban areas related to environmental risks. These risks can be somehow quantified using remote sensed, and especially hyperspectral, data.

Indeed, there are already some examples of urban hyperspectral data analysis. In Marino et al. (2000) the authors present urban material characterization, while in Xiao et al. (1999) vegetation canopies and tree analysis in a so called «urban forest» is addressed. Land cover classification and sealed parts detection in urban areas are considered in Segl et al. (2000), Heiden et al. (2001), Roessner et al. (2001a). In all of these papers the need for hyperspectral data for cor- 
rectly accomplishing these tasks is clear, and the reasons may be summarized as follows:

- Imaging spectrometry helps to characterize urban sealing and soil composition, providing in a unique way information useful to define the risks of urban runoff and flooding.

- Hyperspectral imaging may allow identifying and cataloguing potentially dangerous construction materials that have been used for coverings in the past (e.g., asbestos concrete).

- Hyperspectral data acquired in the thermal infrared are useful in studies of urban heat effects, to establish the correlation between building material composition, heat flux and ground temperature.

Our project aims at producing detailed land cover maps exploiting the potentials of hyperspectral mapping, since it has been demonstrated (Roessner et al., 2001b) that significant improvements in classification of urban areas could be achieved using the spectral surface characteristics in the visible and near-infrared range, with a suitable spectral resolution. More in detail, we want to propose precise and useful algorithms for:

- Characterization and classification of land cover in urban areas using supervised approaches.

- Correlation of the spectral characteristics of urban cover materials with their geometry and illumination conditions.

- Definition of the vegetation status and corresponding risk maps for air pollution.

- In summary, medium and high-resolution characterization of urban environment that includes built structures, road networks, etc.

Finally, it is worth noting that recent technical studies show that more refined results can be obtained by combining hyperspectral measurements with those from different, complementary sensors or data sets (Madhok and Landgrebe, 1999) or by combining hyperspectral and radar measurements (Gamba and Houshmand, 2001; see also Nevatia et al., 1999).

So, to make the study more effective, hyperspectral data were collected over an urban area where a multi-sensor data set is already available (ERS, SIR-C/X-SAR, Landsat, IKONOS, EROSA1, as well as multitemporal aerial data). An integrated system has been designed, and is currently under extensive testing, to manage this rather large data set (Costamagna et al., 2001).
Data used in this work come from the EU funded HySenS project, proposed by the DLR (German Aerospace Agency) to make the access to research infrastructures easier. In this frame, free access to the data from DLR's airborne imaging spectrometers DAIS 7915 (Digital Airborne Imaging Spectrometer) and ROSIS (Reflective Optics System Imaging Spectrometer) were offered to the European remote sensing community. Imaging spectrometer data sets acquired over test areas proposed by the individual user groups were system corrected and calibrated to at-sensor radiance.

DAIS and ROSIS have a spectral resolution capable of delineating the highly complex land uses, covers and patterns of urban environments, uncovering environmental degradation, such as vegetation stress and soil/artificial materials decomposition. However, environmental urban analysis is a new development in remote sensing (Ben-Dor et al., 1998), for which suitable analysis tools are required.

In the following we will show a more detailed description of the collected data set, the methodology implemented in the project, and some preliminary results obtained with DAIS data, referring to supervised classification approaches, exploiting spectral as well as spatial information.

\section{Data set}

The data used in this work correspond to the records of four flight lines over the urban area of Pavia, Northern Italy, acquired in the summer of 2002. The area was imaged by means of both DAIS and ROSIS, with different spatial and spectral resolution. The flight altitude was chosen as the lowest available for the airplane (about $1500 \mathrm{~m}$ ), which resulted in the finest spatial resolution, i.e. 2.6 and $1.2 \mathrm{~m}$ for DAIS and ROSIS, respectively. The lines were chosen so that the higher resolution sensor (ROSIS) covered the whole urban area. Therefore, the images from the DAIS sensor are partially overlapping, which will allow studying the effects of the directional reflectance of urban materials on mapping accuracy.

Unfortunately, due to unprecedented problems with the recorder of navigation parame- 
ters, no ROSIS data was provided so far to our team and thus only DAIS data were analysed.

During the flight campaign, many ancillary data were collected, and in particular a detailed inspection of the materials in portions of the urban area was performed. Ground spectrometer and sun spectrometer data were collected as a support for the atmospheric correction procedure (relying on MODTRAN), which requires the availability of both ground and at-sensor spectra for a number of land cover cases. Only atmospherically corrected data were then used in this work.

\section{Methodology}

Accurate georeferencing is required to make maps obtained from high spatial resolution data useful for comparison with other data sources and for integration into a GIS. For this reason one of the efforts of this work has been the choice of an algorithm to obtain such a result.

The work was then devoted to information extraction from the HySenS imagery using different approaches for urban and non-urban areas, whose results were then combined.

The main reason for processing the data in two parts is that the characteristics of scenes are very different. For the urban part, spatial structure plays a very important role in discrimination. For classification of the non-urban area, the spectral information is of prime importance.

The main features were extracted from both data sets using two well established extraction methods, namely Discriminant Analysis Feature Extraction (DAFE) and Decision Boundary Feature Extraction (DBFE) (Landgrebe, 2003). Classification algorithms were then applied to both the original data and the extracted features.

For urban areas, the neuro-fuzzy spectral+ +spatial approach outlined in Gamba and Dell'Acqua (2003) was followed, and results compared with that of classical classification algorithms.

For non-urban areas, instead, the data were assumed to be Gaussian distributed. A very limited number of labeled training samples is available, as the scene is mainly urban. Therefore, methods based on the use of enhanced statistics

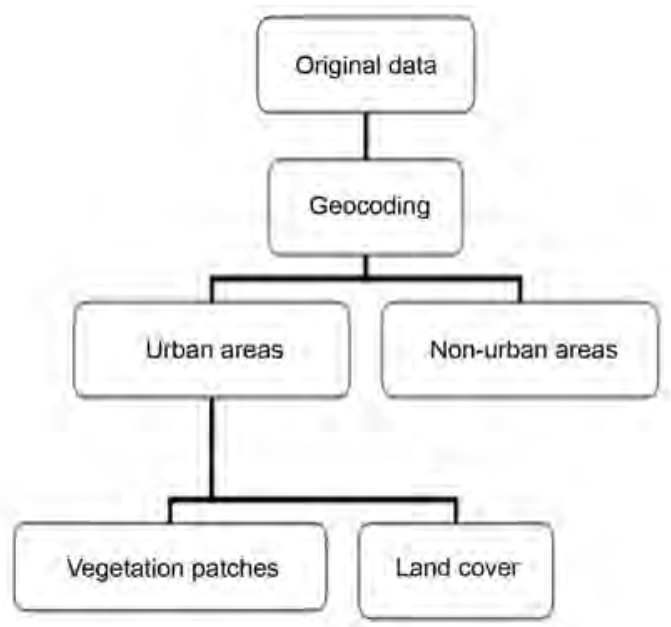

Fig. 1. The work flow of the research presented in this paper.

(Landgrebe, 2003) were applied. The enhanced statistics methods use some unlabeled samples to improve the estimates of the parameters used in the classification. The maximum likelihood classifier was used for classification.

The methodology applied may be summarized in fig. 1, and corresponds to the structure of this paper, where the next section will be devoted to geocoding issues, while Section 5 will provide some examples of classification results exploiting spatial as well as spectral characteristics of the urban environment.

For the vegetation, NDVI was calculated over the entire image after averaging red and IR bands; the NDVI image was then processed to extract the Weighted Mean Patch Size (WMPS) index and the Lacunarity index which were then used to characterize the distribution of vegetation inside the considered urban area.

\section{Geocoding}

To geocode the DAIS images, two nonparametric methods were experimented. A polynomial geometric correction was carried out using Ground Control Points (GCPs) selected manually from 1:2000 vector Topographic Maps and a polynomial cubic function. For the 
flight line 2 we used 28 GCPs, distributed over the whole area obtaining the average RMS error of 1 pixel. The same procedure was followed for the flight line 3 with 36 GCPs resulting in an average RMS error of 0.91 pixel.

To improve the accuracy another method was tested. The software PCI OrthoEngine 8.2 Satellite Project was used to carry out geometric corrections based on rational functions (see Dowman and Dolloff, 2000), where the relation between ground points and corresponding image points is defined as a ratio of polynomials. No system data is needed, but a DEM is necessary, as well as a minimum number of GCPs, related to the degree of the polynomial employed.

We performed the geocoding of the image line 3 using 33 GCPs and a polynomial of the 2nd order, resulting in an average RMS error in $X$ pixel coordinate of about 0.26 and $Y$ pixel coordinate of about 0.27 .

The rectified images (line 2 and line 3 ) were resampled using nearest neighbour so as to retain the spectral scale values for subsequent multispectral classification.

\section{Urban mapping}

After geocoding, urban land cover mapping and classification was considered. As a preliminary step, an analysis of the spectra of materials commonly found in the urban area was performed, using CNR spectral library. As a consequence, after proper radiometric and atmospheric calibration (based on MODTRAN, Berk et al., 1989), and taking into account the spatial ground dimension of the DAIS pixel, nine cover classes were considered, namely water, tree, asphalt, parking (asphalt with some concrete and soil), bitumen, bricks, meadow, bare soil, shadow. More refined classes are expected to be found after proper analysis of the results of this preliminary processing step of the data set.

\subsection{Spectral and spatial land cover mapping}

To provide a quantitative evaluation of the classification results, a sample of the data was considered (fig. 2), and ground truth was pro-

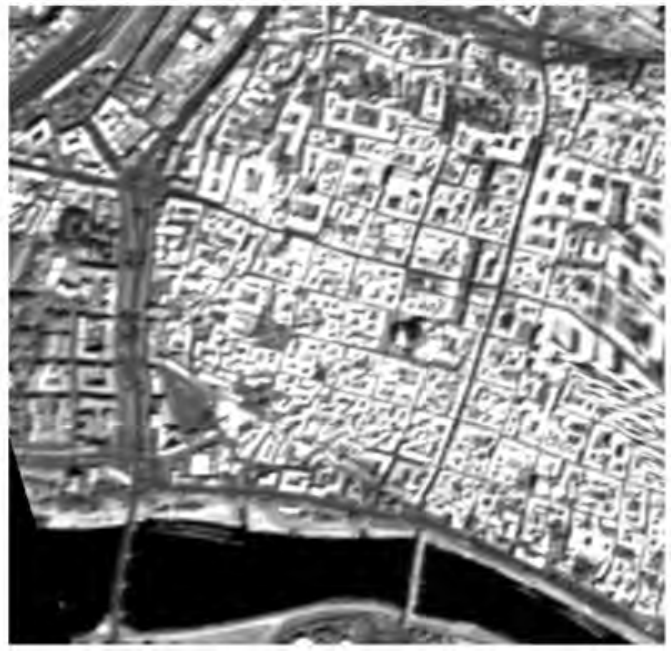

Fig. 2. A sample image of the test area used for classification, near the town center (north is to the bottom); this image was obtained by averaging several channels in the visible range of wavelengths.

vided by available high resolution aerial and satellite images and with a ground survey.

As already mentioned in the previous section, a complete analysis of the urban land cover cannot be obtained without considering both a spectral and a spatial characterization. Therefore, we applied either on the original data set or on the one reduced by means of the DAFE or BDFE approaches, the neurofuzzy classification chain introduced in Gamba and Dell'Acqua (2003). This is a two step approach, considering first a spectral analysis of the image with a neural approach (spectral ARTMAP), and followed by a re-processing step taking into account the spatial patterns of the first classification map (spatial ARTMAP). The spectral analysis using DBFE processed data provided an overall accuracy of $94.3 \%$, while the data coming from DAFE feature extraction process provided a value of $96.7 \%$. After the second step, these values were enhanced to $95.3 \%$ and $97 \%$, respectively. See a visual comparison of spectral and spatial classification results in fig. 3a,b and table I.

Other supervised classification methods were tested on the same area. Results, in terms 

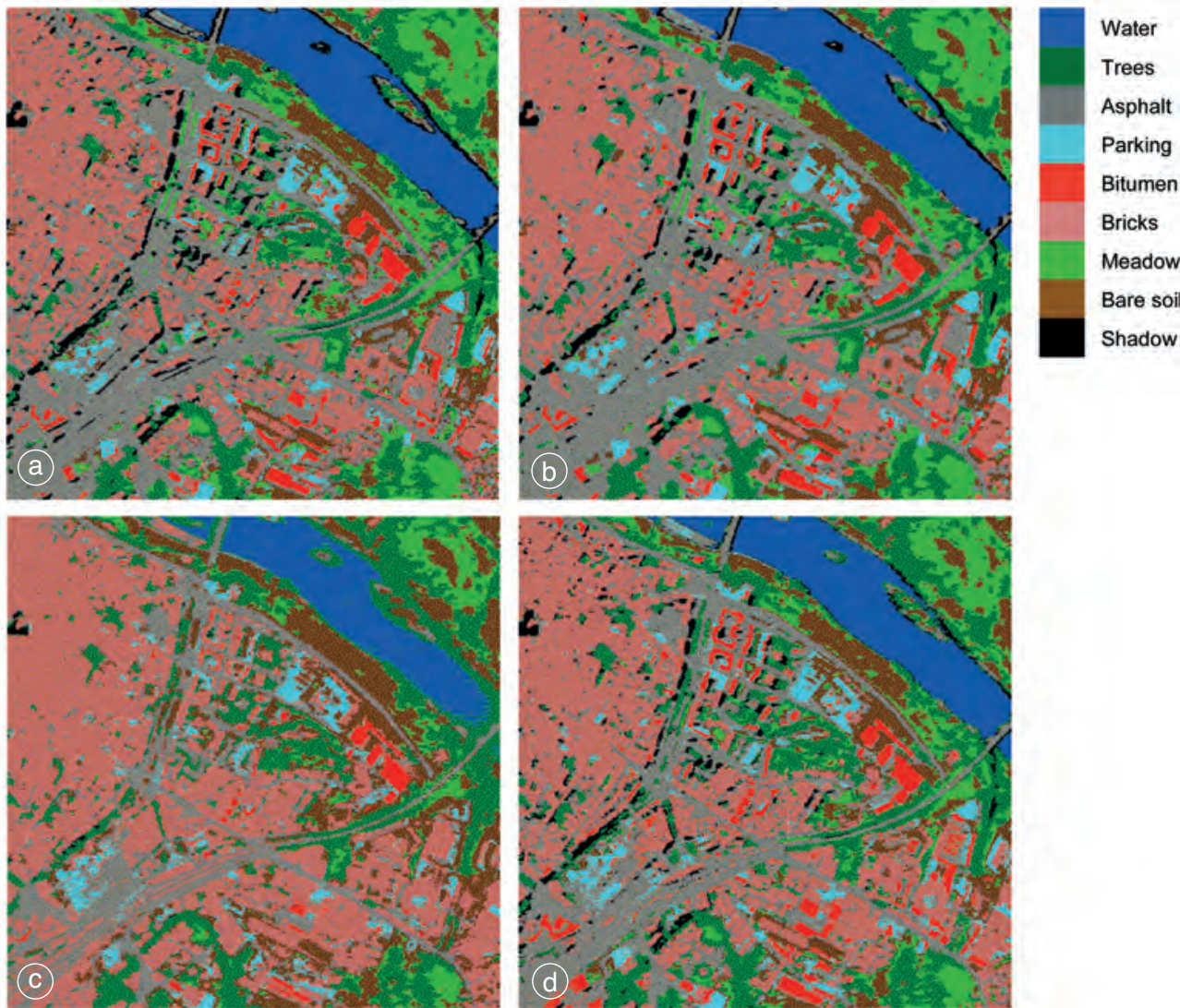

Fig. 3a-d. Classification maps for a) the spectral ARTMAP and b) spatial ARTMAP classifier applied to the data after a DAFE analysis; classification maps for c) the ECHO and d) Maximum Likelihood classifier applied to the data after a DAFE analysis.

Table I. Accuracy values for ARTMAP applied to the test area in fig. 2.

\begin{tabular}{ccccc}
\hline \hline & \multicolumn{2}{c}{ Spectral ARTMAP } & \multicolumn{2}{c}{ Spatial ARTMAP } \\
& Producer's accuracy & User's accuracy & Producer's accuracy & User's accuracy \\
\hline Water & $100 \%$ & $100 \%$ & $100 \%$ & $100 \%$ \\
Trees & $93.6 \%$ & $98.8 \%$ & $94.2 \%$ & $98.5 \%$ \\
Asphalt & $98.6 \%$ & $96.9 \%$ & $98.8 \%$ & $96.6 \%$ \\
Parking & $96.9 \%$ & $99.9 \%$ & $90.9 \%$ & $92.6 \%$ \\
Bitumen & $99.5 \%$ & $99.9 \%$ & $97.7 \%$ & $99.7 \%$ \\
Bricks & $92 \%$ & $87.7 \%$ & $99.4 \%$ & $99.9 \%$ \\
Meadow & $99.4 \%$ & $82.7 \%$ & $99.5 \%$ & $82.6 \%$ \\
Bare soil & $86.9 \%$ & $98.3 \%$ & $87.6 \%$ & $98.1 \%$ \\
Shadow & $90.9 \%$ & $81.1 \%$ & $87.3 \%$ & $88.5 \%$ \\
\hline
\end{tabular}


Table II. Overall accuracy values for the test area in fig. 2.

\begin{tabular}{cccc}
\hline \hline & Original data & DBFE & DAFE \\
\hline Maximum Likelihood & $95.6 \%$ & $96.3 \%$ & $97.6 \%$ \\
Fisher & $97 \%$ & $96.7 \%$ & $97 \%$ \\
Minimum distances & $73.6 \%$ & $87 \%$ & $96.6 \%$ \\
ECHO & $95.6 \%$ & $96.3 \%$ & $97.6 \%$ \\
SAM with 0.05 rad & $87.4 \%$ & $=$ & $=$ \\
\hline
\end{tabular}

Table III. Accuracy values for Maximum Likelihood classifier applied to the test area in fig. 2.

\begin{tabular}{ccc}
\hline \hline & Producer accuracy & User accuracy \\
\hline Water & $100 \%$ & $100 \%$ \\
Trees & $95.2 \%$ & $98.3 \%$ \\
Asphalt & $96.2 \%$ & $98.9 \%$ \\
Parking & $91.6 \%$ & $82.2 \%$ \\
Bitumen & $98.3 \%$ & $99.4 \%$ \\
Bricks & $99.8 \%$ & $99.2 \%$ \\
Meadow & $98.4 \%$ & $90.1 \%$ \\
Bare soil & $94.3 \%$ & $97.7 \%$ \\
Shadow & $89.2 \%$ & $82.7 \%$ \\
\hline
\end{tabular}

of classification accuracy, are shown in table II. The Spectral Angle Mapper (SAM) obtained poor results; this is probably due to the choice of the training set, optimized for statistical methods but unsuitable for SAM which works best on pure spectra instead.

Figure $3 \mathrm{c}, \mathrm{d}$ and table III present the results for the Maximum Likelihood and ECHO classifiers applied after the DAFE feature extraction procedure. Table III reports the best of the two results, i.e. the one given by the Maximum Likelihood classifier.

\subsection{Urban spatial structure investigation}

Together with accurate urban land cover maps, among the possible spatial characterizations of an urban environment there is a growing need for quality of life indicators. In particular, the availability and distribution of green areas is a good indicator of both the air quality (which heavily affects the quality of life, especially in urban centers) and the possibility to have openair entertainment areas. The approach taken in this work is to characterize the distribution of patches of vegetation in urban areas.

As a first processing step, the pixels in our DAIS data set were classified into vegetative or non-vegetative pixels using the Normalized Difference Vegetation Index (NDVI, Tucker and Sellers, 1986). The red bands 7 to 12 (0.63 to 0.70 $\mathrm{mm})$ were averaged to make a red image, and the near infrared bands 16 to 21 (0.76 to $0.85 \mathrm{~mm})$ were averaged to make a near infrared image.

The NDVI image was then thresholded to create an image that contains patches of vegetative pixels. These patches were then analyzed using two metrics: a weighted mean patch size metric and a Lacunarity metric.

The Weighted Mean Patch Size (WMPS) index (Li and Archer, 1997) calculates the patch area weighted by the number of patches in the scene possessing that area. An image was derived which shows the WMPS calculated over a local window within the image. A window is moved across the image in a raster scan fashion, and the WMPS is assigned to the central pixel in each window. The WMPS image thereby shows the spatial distribution of patches within the image.

The Lacunarity index (Plotnick et al., 1993) shows the variability of size of vegetated patches, and is an indicator of «clumpiness». In a way similar to the WMPS, it has been applied to calculate a single value for each point in the scene. The Lacunarity index has an additional parameter known as the box size, which results in a Lacunarity image for each box size; results are shown in fig. $4 \mathrm{a}-\mathrm{d}$.

These experiments should help provide new insights into landscape structure, which can be exploited in land use planning and in the creation of heuristics for planning sustainable urban development.

Thresholding the Normalized Difference Vegetation Index (NDVI) pixels are classified into vegetative or non-vegetative pixels. The quality of the Lacunarity and Weighted Mean Patch Size (WMPS) metrics is determined by the robustness of this classification. The upper 

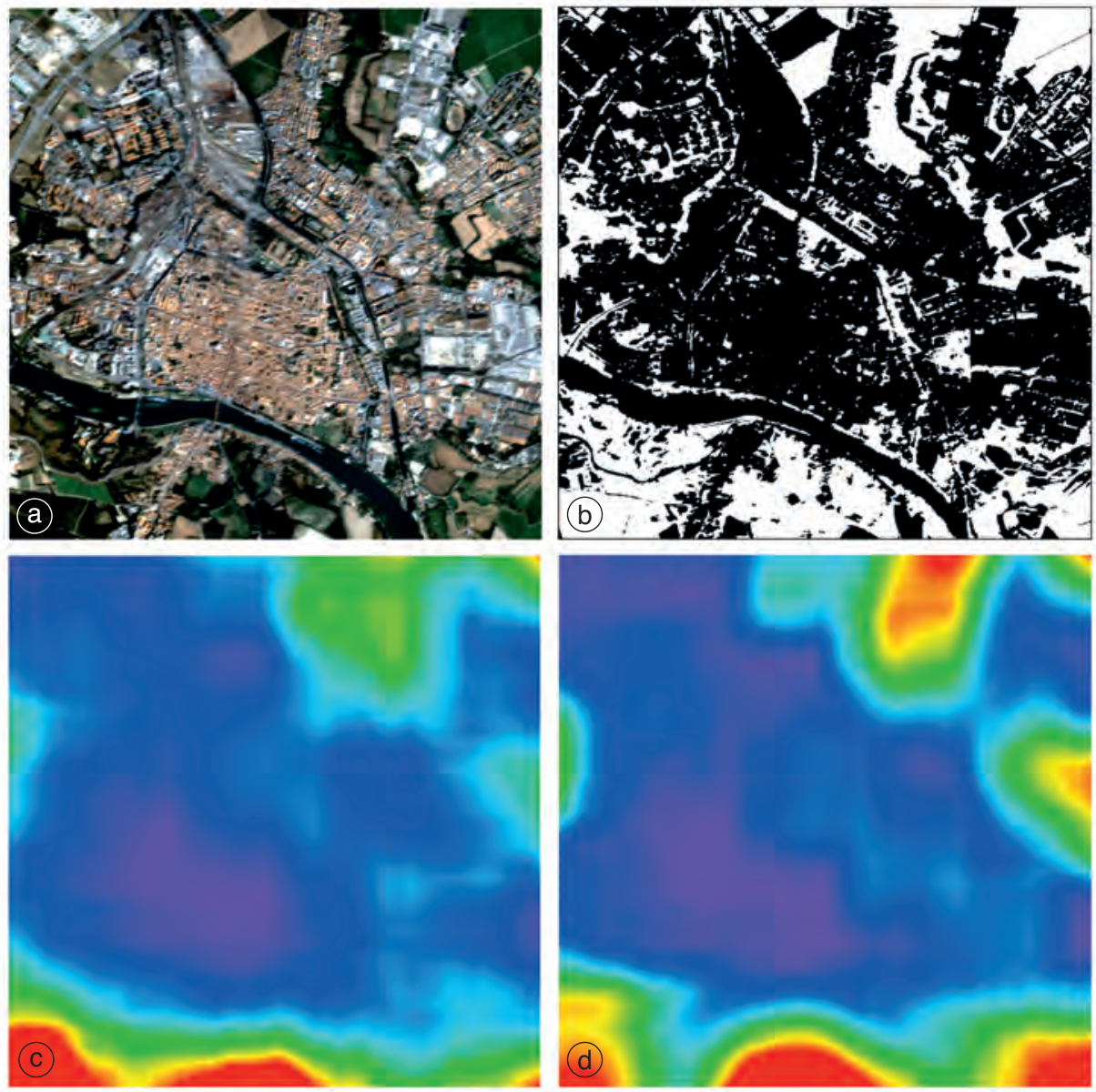

Fig. 4a-d. a) The area of interest; b) NDVI image of the same area, thresholded at 0.5; c) Lacunarity image (window size 251, box size 9); d) WMPS image (window size 251).

and lower areas of the image (fig. 4a-d) contain the most vegetative pixels (displayed in white in fig. 4b). These regions also have the highest Lacunarity (fig. 4c) and WMPS (fig. 4d). The centre of the region is mostly urban, and has low values for both metrics.

A large number of ecological metrics are available and it is important to establish which are the most useful. Early results from this work appear to suggest that for environmentally sustainable land use in sub-urban areas local WMPS should be maintained at values equivalent to $0.16 \mathrm{~km}^{2}$ and Lacunarity at values ex- ceeding 2.0 at box size $28 \times 28 \mathrm{~m}$ and exceeding 1.4 at box size $204 \times 204 \mathrm{~m}$. These values are intended to ensure that a relatively low density of housing is maintained with a good clustering of local green areas, which would be supportive of recreation and diversification of flora and fauna.

\section{Conclusions and future work}

This work shows that it is possible to extract from hyperspectral data very important infor- 
mation, useful for the environmental characterization of urban areas. Even if in its preliminary stages, this research has shown many potentialities for urban remote sensing.

The co-registration of overlapping areas acquired by the DAIS in parallel flight lines having opposite directions opens the possibility to characterize urban materials considering also directionality effects. This study will be helped by a number of precise ground reflectance measurements, made available thanks to CNR efforts.

\section{Acknowledgements}

The authors wish to thank the DLR for performing the campaign and providing the data within the framework of the HySenS project and the CNR for collecting the ground reflectance data and the atmospheric calibration data and for their support.

\section{REFERENCES}

Ben-Dor, E., N. LEVIN and H. SAARONI (1998): Utilization of imaging spectrometry in urban areas: a case study over Tel-Aviv, Israel using the CASI sensor, in Proceedings of the 1st EARSeL Workshop on Imaging Spectrometry, Zurich, Switzerland, 473-479.

BerK, A., L.S. BERNSTEIN and D.C. RoBERTSON (1989): MODTRAN: a moderate resolution model for LOWTRAN 7 (Phillips Lab., Hanscom AFB, MA), Tech. Rep. GL-TR-89-0122.

Costamagna, E., P. Gamba and V. Casella (2001): Environmental applications of the MURA DI PAVIA project, in Proceedings of the International Workshop on Geo-Spatial Knowledge Processing for Natural Resource Management (CD-ROM).

DowmAn, I. and J.T. DOLlofF (2000): An evaluation of rational functions for photogrammetric restitution, in IAPRS, Amsterdam, vol. XXXIII.

Gamba, P. and F. Dell'ACQuA (2003): Improved multiband urban classification using a neuro-fuzzy classifier, Int. J.Remote Sensing, 24 (4), 827-834.

GAmBA, P. and B. Houshmand (2001): Integration of AVIRIS and IFSAR data for improved 3D urban profile reconstruction, Photogramm. Eng. Remote Sensing, 67 (8), 947-956.
Heiden, U., S. Roessner, K. Seg and H. Kaufmann (2001): Analysis of spectral signatures of urban surfaces for area-wide identification using hyperspectral HyMap data, in Proceedings of the IEEE/ISPRS Joint Workshop on Data Fusion and Urban Remote Sensing over Urban Areas, Rome, Italy, 173-176.

Landgrebe, D.A. (2003): Signal Theory Methods in Multispectral Remote Sensing (John Wiley and Sons, Hoboken, New Jersey).

LI, B.-L. and S. ARCHER (1997): Weighted mean patch size: a robust index for quantifying landscape structure, Ecol. Modelling, 102, 353-361.

MAdHoK, V. and D. LANDGREBE (1999): Supplementing hyperspectral data with digital elevation, in Proceedings of the International Geoscience and Remote Sensing Symposium (IGARSS '99), Hamburg, Germany, June 28-July 2, 1999, IEE 1999 International, 1, 59-61, doi: 10.1109/IGARSS.1999.773400.

Marino, C.M., C. Panigada, L. Busetto, A. Galli and M. BosCHETTI (2000): Environmental applications of airborne hyperspectral remote sensing: asbestos concrete sheeting identification and mapping, in Proceedings of the 14th International Conference and Workshops on Applied Geologic Remote Sensing, August 2000, Las Vegas, Nevada, U.S.A., CD-ROM.

Nevatia, R., A. Huertas and Z. KIm (1999): The MURI project for rapid feature extraction in urban areas, in $I S$ PRS: Automatic Extraction of GIS Objects from Digital Imagery, Munich, 3-14.

PlotNICK, R.E., R.H. GARDNER and R.V. O'NeILl (1993): Lacunarity indices as measures of landscape texture, Landscape Ecol., 8, 201-211.

Roessner, S., K. Segl, U. Heiden and H. Kaufmann (2001a): Automated differentiation of urban surfaces based on airborne hyperspectral imagery, IEEE Trans. Geosci. Remote Sensing, 39 (7), 1525-1532.

Roessner S., K. SEgl, U. Heiden and H. Kaufmann (2001b): Analysis of spectral signatures of urban surfaces for their identification using hyperspectral HyMap data, in Proceedings of the IEEE/ISPRS Joint Workshop on Data Fusion and Remote Sensing over Urban Areas, 8-9 November, Rome, Italy, 173-177.

Segl, K., S. Roessner and U. HeIden (2000): Differentiation of urban surfaces based on hyperspectral image data and a multi-technique approach, in Proceedings of the IEEE Geoscience and Remote Sensing Symposium, Honolulu, vol. 4, 1600-1602.

Tucker, C.J. and P.J. SEllers (1986): Satellite remote sensing of primary production, Int. J. Remote Sensing, 7, 1395-1416.

Xiao, Q., S.L. Austin, E.G. McPherson and P.J. PePer (1999): Characterization of the structure and species composition of urban trees using high resolution AVIRIS data, in Proceedings of the AVIRIS Workshop, Pasadena, CA. 